\title{
A preliminary examination of gut microbiota, sleep, and cognitive flexibility in healthy older adults
}

\author{
Jason R. Anderson a , Ian Carroll ${ }^{\mathrm{b}}$, M. Andrea Azcarate-Peril ${ }^{\mathrm{c}}$, Amber D. Rochette ${ }^{\mathrm{a}}$, \\ Leslie J. Heinberg ${ }^{\mathrm{d}}$, Christine Peat ${ }^{\mathrm{e}}$, Kristine Steffen ${ }^{\mathrm{f}}$, Lisa M. Manderino ${ }^{\mathrm{a}}$, \\ James Mitchell ${ }^{\text {, h }}$, John Gunstad ${ }^{\text {a, * }}$ \\ a Department of Psychological Sciences, Kent State University, 600 Hilltop Drive, Kent, OH, 44242, USA \\ ${ }^{\mathrm{b}}$ Department of Medicine, University of North Carolina at Chapel Hill, 321 S Columbia Street, Chapel Hill, NC, 27516, USA \\ ${ }^{\mathrm{c}}$ Department of Medicine and Microbiome Core Facility, University of North Carolina School of Medicine, 321 S Columbia Street, Chapel Hill, NC, 27516, USA \\ d Cleveland Clinic, Lerner College of Medicine of Case Western Reserve University, Bariatric and Metabolic Institute, 9500 Euclid Ave, Cleveland, OH, 44195, \\ USA \\ e Department of Psychiatry, University of North Carolina School of Medicine, 321 S Columbia Street, Chapel Hill, NC, 27516, USA \\ ${ }^{\mathrm{f}}$ Department of Pharmaceutical Sciences, College of Pharmacy, Nursing and Allied Sciences, North Dakota State University, PO Box 6050, Fargo, ND, 58102, \\ USA \\ ${ }^{g}$ Neuropsychiatric Research Institute, 120 South 8th Street, Fargo, ND, 58107, USA \\ h Department of Psychiatry and Behavioral Science, School of Medicine and Health Services, University of North Dakota, 1919 Elm Street North, Fargo, ND, \\ 58102, USA
}

\section{A R T I C L E I N F O}

Article history:

Received 3 May 2017

Received in revised form

22 July 2017

Accepted 24 July 2017

Available online 2 August 2017

Keywords:

Sleep quality

Gut microbiome

Aging

Cognitive function

Executive function

Cognitive flexibility

\section{A B S T R A C T}

Objectives: Inadequate sleep increases the risk for age-related cognitive decline and recent work suggests a possible role of the gut microbiota in this phenomenon. Partial sleep deprivation alters the human gut microbiome, and its composition is associated with cognitive flexibility in animal models. Given these findings, we examined the possible relationship among the gut microbiome, sleep quality, and cognitive flexibility in a sample of healthy older adults.

Methods: Thirty-seven participants (age $64.59 \pm 7.54$ years) provided a stool sample for gut microbial sequencing and completed the Pittsburgh Sleep Quality Index and Stroop Color Word Test as part of a larger project.

Results: Better sleep quality was associated with better Stroop performance and higher proportions of the gut microbial phyla Verrucomicrobia and Lentisphaerae. Stroop Word and Color-Word performance correlated with higher proportions of Verrucomicrobia and Lentisphaerae. Partial correlations suggested that the relationship between Lentisphaerae and Stroop Color-Word performance was better accounted for by sleep quality; sleep quality remained a significant predictor of Color-Word performance, independent of the Lentisphaerae proportion, while the relationship between Lentisphaerae and Stroop performance was non-significant. Verrucomicrobia and sleep quality were not associated with Stroop Word performance independent of one another.

Conclusions: The current findings suggest a possible relationship among sleep quality, composition of the gut microbiome, and cognitive flexibility in healthy older adults. Prospective and experimental studies are needed to confirm these findings and determine whether improving microbiome health may buffer against sleep-related cognitive decline in older adults.

\footnotetext{
* Data collected at Kent State University. Gut microbiome sequencing completed at the University of North Carolina.

* Corresponding author. Fax: +1330 6723786.

E-mail address: jgunstad@kent.edu (J. Gunstad).
}

\section{Introduction}

Poor sleep often presages age-related cognitive decline and neurodegeneration [1]. The gut microbiota-the community of microorganisms in the gut - may play a role in these phenomena as they are implicated in several risk factors for the development of 
dementia (eg, obesity, diabetes, cardiovascular dysfunction) [2-4]. A recent study found that recurrent sleep restriction alters microbiome composition in healthy, young adult males [5]. Work in animal models also shows that diet-driven changes in microbiome composition can lead to reduced cognitive flexibility [6]. These findings together indicate that dysbiosis of the gut microbiome (atypical composition/diversity) may contribute to the cognitive dysfunction associated with chronically poor sleep.

The current preliminary study examines the possible association between the gut microbiome composition and sleep quality in healthy older adults and whether sleep quality and microbial phyla are independently associated with a measure of cognitive flexibility.

\section{Methods}

\subsection{Participants}

Thirty-seven English-speaking participants aged from 50 to 85 years underwent cognitive testing, completed questionnaires, and provided a stool sample for gut microbiome sequencing. Exclusion criteria included history of neurological, developmental, or severe psychiatric disorder (eg, dementia, stroke, schizophrenia), antibiotic or probiotic use within 30 days of participation in the study; history of significant gastrointestinal disorder or surgery (eg, gastric bypass, Crohn's disease); history of alcohol or illicit drug dependence, and history of severe heart, kidney, or liver problems.

\subsection{Measures}

\subsubsection{Sleep quality}

The Pittsburgh Sleep Quality Index (PSQI) [7] assessed selfreported sleep quality (eg, sleep duration, onset latency) over the past month. The global score served as the outcome of interest, with higher scores indicating poorer sleep quality.

\subsubsection{Cognitive flexibility}

The Stroop Color-Word Test [8] assessed cognitive flexibility. Participants read aloud color words ("Stroop Word"), identified the ink color of rows of X's ("Stroop Color"), and identified the ink color of incongruent color words ("Stroop Color-Word") as quickly as possible. Higher scores indicated more properly identified items over 45 s. Subtest scores were converted to T-scores based on population norms [8].

\subsubsection{Medical questionnaire}

Participants self-reported their current medical conditions, including hypertension, type 2 diabetes mellitus, and sleep apnea.

\subsubsection{Dietary habits}

The EPIC-Norfolk Food Frequency Questionnaire [9] assessed habitual food intake over a 30-day period. The current study assessed macronutrient values-daily grams of protein, sugar, total carbohydrates, and fat and daily energy intake (kcal)-as covariates, given the relationship between diet and the gut microbiome [6].

\subsection{Procedures}

All procedures were approved by the Kent State Institutional Review Board, and all participants provided written informed consent prior to study participation. Participants were recruited from a local community recreation center and scheduled for a single assessment at a time convenient for the individual. Participants underwent testing in a quiet room and were given a stool sample kit and questionnaires to complete at home. Stool samples were collected using prearranged kits from the Ubiome company (www.ubiome.com). The participants mailed their kits directly to Ubiome in sterile capped tubes preserved with proprietary buffer, and 16S rRNA amplicon sequencing was completed following protocols from the Human Microbiome Project [10]. Gut microbiome composition was represented as the proportion each phylum in the gut microbiota.

\subsection{Statistical analyses}

To identify possible confounding variables, we examined the relationships among medical conditions, macronutrient content, and Stroop performance through correlations. Additional correlations were used to assess the associations among PSQI scores, Stroop performance, and gut microbiome composition. Finally, partial correlations determined whether the association between gut microbiome composition and Stroop test performance was independent of PSQI scores. As parametric statistical tests are precluded when analyzing proportions [11], we used Spearman correlations to examine gut microbial phyla and Pearson correlations for all other analyses. Our sample of 37 participants was powered to detect an $r$ of approximately 0.38 with $\alpha=0.05$ and power $=0.80$.

\section{Results}

\subsection{Demographics and preliminary analyses}

Participants (73\% female; 92\% Caucasian) were a mean age of $64.59 \pm 7.54$ years with a mean PSQI score of $5.00 \pm 3.20$. The mean, standardized performance of the sample (T-scores) for the Stroop Word $(\mathrm{M}=48.51 \pm 6.71)$, Color $(\mathrm{M}=48.30 \pm 6.87)$, and Color-Word subtests $(\mathrm{M}=51.22 \pm 10.22)$ fell within the average range for healthy older adults. See Table 1 for descriptive information and unadjusted correlation results.

Regarding confounding variables, hypertension (27\% of the sample) was associated with performance on the Stroop Word $(r=-0.38, p=0.021)$ and Color $(r=-0.33, p=0.045)$ subtests, and was thus utilized as a covariate in further analyses. No relationship with test performance was found for diabetes (5\%) or sleep apnea (11\%). Several nutritional indices were associated with test performance, including daily averages for energy (1519.24 $\pm 912.00 \mathrm{kcal})$, carbohydrate $(162.09 \pm 111.95 \mathrm{~g})$, protein $(72.19 \pm 28.77 \mathrm{~g})$, fat $(66.66 \pm 46.30 \mathrm{~g})$, and sugar intake $(91.31 \pm 63.67 \mathrm{~g})$. Because of the high multicollinearity among these indices (all $r$ 's $>0.71$ ), only the macronutrient that had the strongest relationship with Stroop performance (ie, carbohydrate intake) was included as a covariate in primary analyses.

\subsection{Relationships among sleep quality, gut microbiota, and cognitive flexibility}

Poor sleep quality was associated with poorer performance on the Stroop Word $(r=-0.40, p=0.018)$ and Color-Word $(r=-0.41$, $p=0.010$ ) subtests after controlling for hypertension and carbohydrate intake, while a trend in the same direction was seen for Stroop Color performance $(r=-0.34, p=0.053)$. Poor sleep quality was also associated with lower proportions of the phyla Verrucomicrobia and Lentisphaerae.

After controlling for carbohydrate intake and hypertension, the proportion of Verrucomicrobia showed a positive correlation with Stroop Word performance $(r=0.36, p=0.034)$ and trended toward a positive correlation with Stroop Color performance $\left(r_{\mathrm{s}}=0.31\right.$, $p=0.071)$. Lentisphaerae was not associated with either Stroop 
Table 1

Gut microbial phyla descriptives $(N=37)$.

\begin{tabular}{|c|c|c|c|c|c|c|c|c|}
\hline \multirow[t]{2}{*}{ Microbial phylum } & \multicolumn{4}{|c|}{ Proportion of total bacteria } & \multirow[t]{2}{*}{ PSQI $r_{\mathrm{s}}$} & \multirow[t]{2}{*}{ Stroop Word $r_{\mathrm{s}}$} & \multirow[t]{2}{*}{ Stroop Color $r_{\mathrm{s}}$} & \multirow{2}{*}{$\begin{array}{l}\text { Stroop } \\
\text { Color-Word } r_{\mathrm{s}}\end{array}$} \\
\hline & Minimum & Mean & Median & Maximum & & & & \\
\hline Euryarchaeota & 0 & $2.41 \times 10^{-4}$ & 0 & $3.53 \times 10^{-3}$ & 0.04 & -0.23 & -0.13 & -0.02 \\
\hline Actinobacteria & $9.95 \times 10^{-2}$ & $9.06 \times 10^{-3}$ & $4.55 \times 10^{-3}$ & $5.43 \times 10^{-2}$ & -0.18 & 0.27 & $0.37^{*}$ & -0.02 \\
\hline Bacteroidetes & 4.76 & $3.16 \times 10^{-1}$ & $3.02 \times 10^{-1}$ & $6.36 \times 10^{-1}$ & 0.08 & -0.20 & -0.31 & $-0.33^{*}$ \\
\hline Chloroflexi & 0 & $1.47 \times 10^{-6}$ & 0 & $5.45 \times 10^{-5}$ & -0.09 & 0.27 & 0.15 & 0.07 \\
\hline Cyanobacteria & 0 & $1.84 \times 10^{-3}$ & 0 & $2.13 \times 10^{-2}$ & -0.20 & 0.32 & -0.10 & 0.14 \\
\hline Elusimicrobia & 0 & $1.66 \times 10^{-4}$ & 0 & $6.13 \times 10^{-3}$ & 0.08 & -0.21 & -0.20 & 0.02 \\
\hline Firmicutes & $1.53 \times 10^{-1}$ & $5.99 \times 10^{-1}$ & $6.11 \times 10^{-1}$ & $9.18 \times 10^{-1}$ & 0.10 & 0.05 & 0.19 & 0.21 \\
\hline Fusobacteria & 0 & $1.36 \times 10^{-4}$ & 0 & $4.26 \times 10^{-3}$ & 0.20 & -0.16 & -0.24 & -0.08 \\
\hline Lentisphaerae & 0 & $6.21 \times 10^{-5}$ & 0 & $7.44 \times 10^{-4}$ & $-0.37^{*}$ & 0.11 & 0.02 & $0.35^{*}$ \\
\hline Proteobacteria & $8.41 \times 10^{-4}$ & $4.19 \times 10^{-2}$ & $1.78 \times 10^{-2}$ & $4.03 \times 10^{-1}$ & 0.11 & -0.14 & -0.11 & -0.13 \\
\hline Spirochetes & 0 & $7.35 \times 10^{-7}$ & 0 & $2.72 \times 10^{-5}$ & -0.09 & 0.27 & 0.15 & 0.07 \\
\hline Synergistetes & 0 & $6.25 \times 10^{-5}$ & 0 & $1.83 \times 10^{-3}$ & -0.17 & 0.08 & 0.08 & 0.15 \\
\hline TM7 & 0 & $1.23 \times 10^{-5}$ & 0 & $1.01 \times 10^{-4}$ & -0.14 & 0.02 & -0.002 & -0.02 \\
\hline Tenericutes & 0 & $9.26 \times 10^{-4}$ & 0 & $1.03 \times 10^{-2}$ & -0.21 & 0.002 & -0.03 & 0.20 \\
\hline Verrucomicrobia & 0 & $3.10 \times 10^{-2}$ & $6.81 \times 10^{-3}$ & $4.03 \times 10^{-1}$ & $-0.52^{* *}$ & 0.37 & $0.34^{*}$ & 0.29 \\
\hline Thermi & 0 & $2.20 \times 10^{-6}$ & 0 & $3.38 \times 10^{-5}$ & 0.06 & 0.007 & 0.26 & -0.16 \\
\hline
\end{tabular}

Note: ${ }^{*}: p<0.05,{ }^{* *}: p<0.01$. PSQI = Pittsburgh Sleep Quality Index.

Word or Color performance; however, greater proportions were associated with Stroop Color-Word performance, independent of the carbohydrate intake $(r=0.41, p=0.015)$.

Partial correlations then examined whether proportions of Verrucomicrobia and Lentisphaerae were associated with Stroop performance, independent of sleep. Sleep quality $\left(r_{s}=-0.24\right.$, $p=0.17)$ and Verrucomicrobia $\left(r_{s}=0.18, p=0.31\right)$ were not independently associated with Stroop Word performance. While poor sleep quality was correlated with poorer Stroop Color-Word performance independent of Lentisphaerae and carbohydrate intake $\left(r_{\mathrm{s}}=-0.34, p=0.045\right)$, the relationship between Lentisphaerae and Color-Word performance was attenuated and reduced to a trend $\left(r_{\mathrm{s}}=0.28, p=0.098\right)$.

\section{Discussion}

Findings from this preliminary study suggest an association among poor sleep, gut microbiome composition, and cognitive flexibility. Our findings show that lower proportions of Verrucomicrobia and Lentisphaerae are associated with poor sleep quality, raising the possibility that they contribute to metabolic dysfunction and obesity, which are commonly observed in populations with disrupted sleep (see Arble et al., 2015 [12]). Although previous work suggests that increased carbohydrate consumption after poor sleep may also play a role [13], the relationship between poor sleep and microbiome composition was maintained in our study despite controlling for carbohydrate intake. Lower proportions of Verrucomicrobia are observed in prediabetes and increased proportions are seen following dieting and gastric bypass [2]. Similarly, lower proportions of Lentisphaerae are associated with greater weight gain in cattle [14]. However, the current results are in contrast with that obtained by Benedict and colleagues [5], who showed that partial sleep deprivation alters the proportion of phyla other than Verrucomicrobia and Lentisphaerae, suggesting a need for further research in this area.

When considered alongside previous studies, the current results suggest altered gut microbiome composition as a possible mechanism linking inadequate sleep to poor neurocognitive outcomes. The relationship between the gut microbiome and cognitive flexibility was insignificant after accounting for prior sleep history, suggesting that poor sleep results in both poorer cognitive flexibility and altered microbiome composition in older adults. Brief sleep restriction is sufficient to alter microbiome composition even in healthy young adults [5]. Work in rodent models has shown that alterations in gut microbiome composition affect cognitive flexibility [6], learning and memory [15], and the deposition of $\beta$-amyloid in the cortex $[6,16]$. As cross-sectional assessments can distort mediational relationships [17], prospective studies are necessary to determine whether altered microbiome composition mediates the relationship between sleep and cognitive decline [1]. Such work will inform whether probiotics, which improve gut health [18], may buffer against sleep-related cognitive dysfunction.

These findings must be considered along with the limitations of the study. Although informative, the observational nature of our preliminary study precludes causal conclusions. Including multiple measures of cognitive flexibility in future studies would improve measurements and strengthen conclusions [19]. Other sleep measures (eg, actigraphy, sleep diaries) in prospective studies may provide more detailed information than the PSQI. Similarly, screening measures (eg, STOP-Bang) may detect undiagnosed sleep-disordered breathing, an important concern given its potential relationship with cognitive function [20]. Further study in larger samples is also needed to determine the most relevant taxonomic rank for these analyses (eg, phylum, genus). Although the effect sizes reported in this preliminary study are robust, statistical adjustment for multiple comparisons was not utilized given the study's exploratory nature and the novelty of this line of work; larger samples in future studies should accommodate such adjustment. Finally, possible mechanisms for the relationship among sleep, cognitive flexibility, and gut microbiome composition (eg, systemic inflammation) were not examined in this study, and their inclusion would benefit future studies.

\section{Conclusions}

To conclude, this preliminary study demonstrated relationships among sleep quality, composition of the gut microbiome, and cognitive flexibility in healthy older adults. Further study is needed to clarify these relationships and identify possible treatment options.

\section{Funding}

This research did not receive any specific grant from funding agencies in the public, commercial, or not-for-profit sectors. 


\section{Acknowledgements}

The authors would like to acknowledge the contribution of the staff and participants of the EPIC-Norfolk Study. EPIC-Norfolk is supported by the Medical Research Council program grants (G0401527, G1000143) and Cancer Research-UK program grant (C864/A8257). The Microbiome Core Facility is supported in part by the NIH/NIDDK grant P30 DK34987.

\section{Conflict of interest}

The ICMJE Uniform Disclosure Form for Potential Conflicts of Interest associated with this article can be viewed by clicking on the following link: http://dx.doi.org/10.1016/j.sleep.2017.07.018.

\section{References}

[1] Musiek ES, Holtzman DM. Mechanisms linking circadian clocks, sleep, and neurodegeneration. Science 2016;354(6315):1004-8.

[2] Barlow GM, Yu A, Mathur R. Role of the gut microbiome in obesity and diabetes mellitus. Nutr Clin Pract 2015;30(6):787-97.

[3] Scheltens P, Blennow K, Breteler MMB, et al. Alzheimer's disease. Lancet 2016;388(10043):505-17.

[4] Shreiner AB, Kao JY, Young VB. The gut microbiome in health and in disease. Curr Opin Gastroenterol 2015;31(1):69-75.

[5] Benedict C, Vogel H, Jonas W, et al. Gut microbiota and glucometabolic alterations in response to recurrent partial sleep deprivation in normal-weight young individuals. Mol Metab 2016;5(12):1175-86.

[6] Magnusson KR, Hauck L, Jeffrey BM, et al. Relationships between diet-related changes in the gut microbiome and cognitive flexibility. Neuroscience 2015;300:128-40.
[7] Buysse DJ, Reynolds III CF, Monk TH, et al. The pittsburgh sleep quality index: a new instrument for psychiatric practice and research. Psychiatry Res 1989;28:193-213.

[8] Golden CJ, Freshwater SM. Stroop color and word test: revised examiner's manual. Wood Dale, IL: Stoelting Co; 2002.

[9] Mulligan AA, Luben RN, Bhaniani A, et al. A new tool for converting food frequency questionnaire data into nutrient and food group values: FETA research methods and availability. BMJ Open 2014;4, e004503.

[10] Human Microbiome Project Consortium. A framework for human microbiome research. Nature 2012;486(7402):215-21.

[11] Chen K, Cheng Y, Berkout O, et al. Analyzing proportion scores as outcomes for prevention trials: a statistical primer. Prev Sci 2016. http://dx.doi.org/ 10.1007/s11121-016-0643-6.

[12] Arble DM, Bass J, Behn CD, et al. Impact of sleep and circadian disruption on energy balance and diabetes: a summary of workshop discussions. Sleep 2015;38(12):1849-60.

[13] Markwald RR, Melanson EL, Smith MR, et al. Impact of insufficient sleep on total daily energy expenditure, foot intake, and weight gain. Proc Natl Acad Sci USA 2013;110(14):5695-700.

[14] Myer PR, Smith TP, Wells JE, et al. Rumen microbiome from steers differing in feed efficiency. PLoS One 2015;10(6), e0129174.

[15] Bruce-Keller AJ, Salbaum JM, Luo M, et al. Obese-type gut microbiota induce neurobehavioral changes in the absence of obesity. Biol Psychiatry 2015;77(7):607-15.

[16] Minter MR, Zhang C, Leone V, et al. Antibiotic-induced perturbations in gut microbial diversity influences neuro-inflammation and amyloidosis in a murine model of Alzheimer's disease. Sci Rep 2016;6:30028.

[17] Maxwell SE, Cole DA. Bias in cross-sectional analyses of longitudinal mediation. Psychol Methods 2007;12(1):23-44.

[18] Marco ML, Heeney D, Binda S, et al. Health benefits of fermented foods: microbiota and beyond. Curr Opin Biotechnol 2017;44:94-102.

[19] Bollen KA, Noble MD. Structural equation models and the quantification of behavior. Proc Natl Acad Sci USA 2011;108(Suppl. 3):15639-46.

[20] Cross N, Lampit A, Pye J, et al. Is obstructive sleep apnoea related to neuropsychological function in healthy older adults? A systematic review and metaanalysis. Neuropsychol Rev 2017. http://dx.doi.org/10.1007/s11065-0179344-6. 